\title{
TYPES OF ALMOST CONTINUITY
}

\author{
B. D. GARRETT AND K. R. KELLUM
}

\begin{abstract}
The differences for properties of the range space $f(X)$ are considered for functions $f: X \rightarrow Y$, where $f$ is almost continuous relative to $X \times Z$, and $f(X) \subset Z$ $\subset Y$. It is shown that if $f$ is allowed to be almost continuous relative to $X \times Z$, where $X$ is a Peano continuum and $Z$ is a locally connected metric space, then $f(X)$ can be any type of subcontinuum of $Z$. This contrasts the known results for the case where $Z=f(X)$ and almost continuity is relative to $X \times f(X)$. Outer almost continuous retracts $(f: X \rightarrow X$ is almost continuous relative to $X \times X)$ and inner almost continuous retracts ( $f: X \rightarrow X$ is almost continuous relative to $X \times f(X)$ ) are defined. Properties of outer almost continuous retracts, including the existence of an outer almost continuous retract $M$ of a fixed point space $X$, where $M$ does not have the fixed point property, are found.
\end{abstract}

Introduction. If $M=Y \cap Z$ and $f$ is a function from $X$ into $M$, it is possible that $f: X \rightarrow Y$ is almost continuous and $f: X \rightarrow Z$ is not. The case when $f: X \rightarrow f(X)$ is almost continuous has received considerable study, particularly if $f(X)$ is an almost continuous retract of $X$. Under the restriction that $X$ is a Peano continuum, for $f$ : $X \rightarrow f(X)$ almost continuous, the image space $f(X)$ has been characterized [6] as "almost Peano" and much is known about an almost continuous retract of this form [1-5]. However, as was pointed out in [1], allowing $f: X \rightarrow Y$ to be almost continuous and $f(X) \subset Y$, the properties of $f(X)$ may be quite different.

The present paper considers the differing properties of $f(X)$ in the cases: (1) when $f: X \rightarrow f(X)$ is almost continuous; and (2) when $f: X \rightarrow Y$ is almost continuous, $f(X) \subset Y$, and $f(X) \neq Y$. Although the image $f(I)$ of an almost continuous function $f: I \rightarrow f(I)$ is "almost Peano" [6], it will be shown, in Theorem 3, that if $M \subset Y$ and $Y$ is a compact locally connected metric space, there is an almost continuous function $f: I \rightarrow Y$ with $f(X)=M$. There are various "types" of almost continuous retractions of a space $X$ to a subset of $X$ [1], but our considerations are restricted to just two of them, which we call "inner almost continuous retractions" and "outer almost continuous retractions" of $X$. The inner almost continuous retractions (where $f: X \rightarrow f(X)$ is almost continuous) have been extensively studied but, until recently [1], the outer almost continuous retractions (where $f: X \rightarrow X$ is almost continuous) have been given little attention.

Conventions. A function $f: X \rightarrow Y$ is a subspace of the product space $X \times Y$ and no distinction will be made between a function and its graph. The letter $I$ denotes

Received by the editors May 12, 1982 and, in revised form, November 12, 1982.

1980 Mathematics Subject Classification. Primary 54C08; Secondary 54F20, 54F25.

Key words and phrases. Almost continuous function, almost continuous retract, Peano continuum, fixed point property, pseudoarc, absolute retract, Hilbert cube. 
the closed unit interval $[0,1]$; when $Z \subset X \times Y$ and $L \subset X$, then $\left.Z\right|_{L}$ is the restriction of $Z$ to $L$; the projection of $Z$ into $X$ is $p_{1}(z)$, and the projection of $Z$ into $Y$ is $p_{2}(z)$; AR means absolute retract; and the space $X$ has the fixed point property if, for any continuous $f: X \rightarrow X$, there is an $x$ in $X$ such that $f(x)=x$.

The various types of almost continuity that result from Definition 1 are no different than those that result from the original definition of an almost continuous function in [8] by Stallings. We state the definition in the following manner so as to make it easier to distinguish between "types" of almost continuous functions. Those differences are quite important, particularly when considering properties of almost continuous retracts.

Definition 1. If each of $X$ and $Y$ is a space, the function $f: X \rightarrow Y$ is almost continuous relative to $X \times Y$ means that, if $D$ is an open set of $X \times Y$ and $f \subset D$, then there is a continuous function $g: X \rightarrow Y$ and $g \subset D$.

In [6] it is shown that a 2nd countable space $M$ is the image of an almost continuous function $f: I \rightarrow M$ relative to $I \times M$ if and only if $M$ is almost Peano. The following theorem shows that things change drastically when we consider almost continuity relative to $I \times Y$, where $M \subset Y$ and $M \neq Y$.

THEOREM 1. Suppose the continuum $M$ is a subspace of a compact locally connected metric space $Y$. There is an almost continuous function $f: I \rightarrow Y$ relative to $I \times Y$ such that $f(I)=M$.

Proof. The function $f$ can be constructed so that, whenever $C$ is a closed subset of $I \times M$ whose projection into $I$ is uncountable, $f$ contains a point of $C$. Assume $f$ is not almost continuous relative to $I \times Y$ and denote by $K$ a blocking set of $I \times Y$ not meeting $f$. (For a description of blocking sets and their properties see any of the references [1-5].)

The space $Y$ is the union of a finite collection of locally connected continua with arbitrarily small diameters [7, Theorem 3, p. 257]. Thus $M$ is the intersection of a decreasing sequence of Peano continua $P_{1}, P_{2}, P_{3}, \ldots$ The blocking set $K$ must intersect each continuous function from $I$ into $P_{1}$ and, therefore, $K$ contains an irreducible blocking set $K_{1}$ of $I \times P_{1}$. Next, $K_{1}$ must intersect each continuous function from $I$ into $P_{2}$ and $K_{1}$ contains an irreducible blocking set $K_{2}$ of $I \times P_{2}$. This process can be continued so that, by induction, there is a decreasing sequence of compact sets $K_{1}, K_{2}, K_{3}, \ldots$ such that for each positive integer $i, K_{i}$ is an irreducible blocking set of $I \times P_{i}$, and, by Lemma 2 of [6], the projection $p_{1}\left(K_{i}\right)$ of $K_{i}$ into $I$ is a closed interval of $I$.

Consider $H=\cap_{i=1}^{\infty} K_{i}$. Clearly $H \subset I \times M$ and $p_{1}(H)$ is closed and connected. Now $f$ does not meet $K$ and therefore does not meet $H$. By the construction of $f$, $p_{1}(H)$ must be a point we will call $z$. Suppose $U$ and $V$ are open sets of $I$ and $Y$, respectively, such that $U \times V$ is an open set of $I \times Y$ containing $(z, f(z))$ and $U \times V$ contains no point of $H$. There is a positive integer $n$ so that $p_{1}\left(K_{n}\right) \subset U$, in which case $K_{n}$ cannot intersect the constant function $g: I \rightarrow P_{n}$ such that $g(x)=f(z)$ for each $x$ in $I$. Since this is a contradiction we have shown that $f$ is almost continuous relative to $I \times Y$ and $f(I)=M$. 
Note. From the literature we know that if $f: X \rightarrow Y$ is almost continuous relative to $X \times f(X)$ and $X$ is a Peano continuum, then those subcontinua of $Y$ which might be $f(X)$ are severely restricted, while under the conditions of Theorem 1, that is not the case. Nevertheless, wthout those requirements on $Y$ we may get similar results for the cases where $f$ is almost continuous relative to $X \times f(X)$ and where $f$ is almost continuous relative to $X \times Y$. Consider what happens when $Y$ is a pseudoarc. It is easy to show that, for either $f: X \rightarrow Y$ almost continuous relative to $X \times f(X)$, or for $f: X \rightarrow Y$ almost continuous relative to $X \times Y$, then $f$ is a constant function.

Definition 2. Suppose $M$ is a subspace of the space $X$ and $f: X \rightarrow M$ is a function such that $f(X)=M$ and $f(x)=x$ for $x$ in $M$. If $f$ is almost continuous relative to $X \times M$, then $f$ is called an inner almost continuous retraction of $X$ and $M$ is an inner almost continuous retract (IACR) of $X$. If $f$ is almost continuous realtive to $X \times X$, then $f$ is called an outer almost continuous retraction of $X$ and $M$ is an outer almost continuous retract (OACR) of $X$.

The following properties are stated without proof but can be shown to be true using variations of known arguments [1-5].

Property 1. An IACR of a Hausdorff space having the fixed point property has the fixed point property.

Property 2. A compact OACR of an AR has the fixed point property.

Property 3. An IACR of $X$ is an OACR of $X$.

The IACRs have been well studied and many of their important properties are known. One of the most basic is stated in Property 1 and is contrasted for the OACRs in Property 2. We do not have an example of a noncompact OACR of an AR without the fixed point property, but clearly, by Property 3 and results in [2], there are noncompact OACRs with the fixed point property. We would conjecture, because of results in [1], that compactness could be eliminated from the statement of Property 2 provided it were replaced with a requirement that allowed a continuous function $g: f(X) \rightarrow f(X)$ to be extended to a continuous $G: X \rightarrow X$. Theorem 2 shows that there are Hausdorff spaces with the fixed point property having OACRs without the fixed point property. Certainly such an OACR cannot be an IACR.

THEOREM 2. There is a plane continuum with the fixed point property having an $O A C R$ that is a continuum without the fixed point property.

Proof. It will be convenient to have the following objects named.

$S \quad$ is the set of points of the form $(x, \sin 1 / x)$, where $0<x<1$, together with the point set $1 \times[-1, \sin 1]$.

$S_{r} \quad$ is the reflection of $S$ through the point $\left(\frac{1}{2},-2\right)$.

$L_{1}$ is is the point set $0 \times[-3,-1]$.

$L_{2}$ is the point set $1 \times[-3,-1]$.

$a_{0}$ is the point $(0,-1)$.

$a_{1}$ is the point $(1,-1)$.

$D$ is the point set $[0,1] \times[-1,1]$.

$N \quad$ is the point $\operatorname{set} \operatorname{cl}(S)$. 
$\begin{array}{ll}M & \text { is } \operatorname{cl}(S) \cup \operatorname{cl}\left(S_{r}\right) \cup L_{1} \cup L_{2} \\ X & \text { is } M \cup D\end{array}$

It is easy to show that $X$ has the fixed point property, and a rotation about $\left(\frac{1}{2},-2\right)$ shows that $M$ does not.

We will show that $M$ is an OACR of $X$. From [3] it follows that $N$ is an IACR of $D$ and by Property 3 it is an OACR of $D$. Take $k: D \rightarrow D$ to be an outer almost continuous retraction of $D$ onto $N$, and define the function $f: X \rightarrow X$ so that $\left.f\right|_{D}=k$ and $f(x)=x$ for $x$ in $X-D$. Now suppose $U$ is any open set of $X \times X$ containing $f$.

For some positive number $\varepsilon<1$, the open $\varepsilon$-neighborhood $b_{0}$ with center $\left(a_{0}, f\left(a_{0}\right)\right)$ is a subset of $U$, and the open $\varepsilon$-neighborhood $b_{1}$ with center $\left(a_{1}, f\left(a_{1}\right)\right)$ is a subset of $U$. Now with $C_{0}$ the interior of the circle in the plane with center $a_{0}$ and radius $\varepsilon / 2$ and with $C_{1}$ the interior of the circle in the plane with center $a_{1}$ and radius $\varepsilon / 2$, take $x_{0}=(0,-(1+\varepsilon / 4))$. Define an open set $U_{1}$ of $X \times X$ such that:

(1) $f \subset U_{1}$;

(2) $U_{1} \subset U$;

(3) $\left.U_{1}\right|_{D} \subset D \times D$;

(4) $\left.U_{1}\right|_{C_{0} \cap X}=\left.b_{0}\right|_{c_{0} \cap X}$; and

(5) $\left.U_{1}\right|_{C_{1} \cap x}=\left.b_{1}\right|_{C_{1} \cap x}$.

Since $N$ is an OACR of $D$, there is a continuous $g: D \rightarrow D$ where $\left.g \subset U_{1}\right|_{D}$. Now we see that both $\left(a_{0}, g\left(a_{0}\right)\right)$ and $\left(x_{0}, f\left(x_{0}\right)\right)$ are in $\left.U_{1}\right|_{C_{0} \cap X}$, so there is a continuous function $s_{0}: 0 \times[-(1+\varepsilon / 4),-1] \rightarrow X$ such that $S_{0} \subset b_{0}, S_{0}\left(a_{0}\right)=g\left(a_{0}\right)$, and $S_{0}\left(x_{0}\right)=f\left(x_{0}\right)$. Similarly there is a continuous function $S_{1}: 1 \times[-(1+\varepsilon / 4),-1] \rightarrow X$ such that $S_{1} \subset b_{1}, S_{1}\left(a_{1}\right)=g\left(a_{1}\right)$, and $S_{1}\left(x_{1}\right)=f\left(x_{1}\right)$.

The function $G: X \rightarrow X$ is defined so that $\left.G\right|_{D}=g,\left.G\right|_{0 \times[-(1+\varepsilon / 4),-1]}=S_{0}$, $\left.G\right|_{1 \times[-(1+\varepsilon / 4),-1]}=S_{1}$, and otherwise $G(x)=x$. It is easy to show that $G: X \rightarrow X$ is continuous and it was constructed so that $G \subset U$. Thus $M$ is an OACR of $X$.

In [5] the absolute IACRs are described and shown to be equivalent to the $\varepsilon$-ARs. Definition 3 defines absolute retracts in the OACR case. For this case it is convenient to take the ARs as the class of containing spaces.

DEFINITION 3. That a continuum $M$ is an absolute outer almost continuous retract (AOACR) means that whenever $M$ is homeomorphic to a subset $M^{\prime}$ of an AR $X$, then $M^{\prime}$ is an OACR of $X$.

THEOREM 3. In order that a continuum $M$ be an $A C A C R$ it is necessary and sufficient that $M$ be homeomorphic to a compact OACR of the Hilbert cube $Q$.

Proof. Since $M$ can be imbedded in $Z$, the necessity follows from Definition 3.

To prove sufficiency suppose $h: M \rightarrow M^{\prime}$ is a homeomorphism of $M$ onto the compact OACR $M^{\prime}$ of $Q$; take $r: Q \rightarrow Q$ to be an outer almost continuous retraction of $Q$ onto $M^{\prime}$, and $s: M \rightarrow M^{\prime \prime}$ is a homeomorphism of $M$ onto $M^{\prime \prime}$, where $M^{\prime \prime}$ is a subset of the $\operatorname{AR} X$. There must be continuous functions $f: X \rightarrow Q$ and $g: Q \rightarrow X$ which are extensions of $h s^{-1}$ and $s h^{-1}$, respectively. Now the function $g r f: X \rightarrow X$ is such that $\operatorname{grf}(X)=M^{\prime \prime}$ and $\operatorname{grf}(x)=x$, for $x$ in $M^{\prime \prime}$. By Propositions 1 and 4 of [8], grf is an outer almost continuous retraction of $X$ onto $M^{\prime \prime}$. 
Corollary. A compact $O A C R$ of an $A R$ is an $A O A C R$.

The proof of Theorem 4 is routine and is omitted.

THEOREM 4. In order that a continuum $M$ be an AOACR it is necessary and sufficient that, if $M \subset Y, Y$ is an $A R$, and $f: X \rightarrow M$ is continuous, where $X$ is a closed subset of a separable metric space $X^{\prime}$, then there is an extension $F$ of $f$ such that $F$ : $X^{\prime} \rightarrow Y$ is almost continuous relative to $X^{\prime} \times Y$ and $F\left(X^{\prime}\right)=M$.

\section{REFERENCES}

1. B. D. Garrett, Almost continuous retracts, General Topology and Modern Analysis. Academic Press, New York, 1980, pp. 229-238.

2. K. R. Kellum, Non-continuous retracts, Studies in Topology, Academic Press, New York, 1975, pp. $255-261$.

3. On a question of Borsuk concerning non-continuous retracts. I, Fund. Math. 87 (1975), 89-92.

4. $ـ$. On a question of Borsuk concerning non-continuous retracts. II, Fund. Math. 92 (1976), $135-140$.

5. _. The equivalence of absolute almost continuous retracts and $\varepsilon$-absolute retracts, Fund. Math. 96 (1977), 229-235.

6. Almost continuous images of Peano continua, Topology and App. 11 (1980), 293-296.

7. K. Kuratowski, Topologv, vol. 2, Academic Press, New York 1968.

8. J. Stallings, Fixed point theorems for connectivity maps, Fund. Math. 47 (1959), 249-263.

Department of Mathematics, Texas A \& M University, College Station, Texas 77843

Department of Mathematics, San Jose State University, San Jose, California 95192 